Proc. Indian Acad. Sci. (Earth Planet. Sci.), Vol. 92, Number 1, March 1983, pp. 45-55.

- Printed in India.

\title{
Indices of equatorial electrojet and counter-electrojet in the Indian region: Evolution of the indices and their authenticity
}

\author{
B N BHARGAVA, B R ARORA and N S SASTRI \\ Indian Institute of Geomagnetism, Colaba, Bombay 400 005, India.
}

MS received 19 February 1981; revised 31 August 1981

\begin{abstract}
Indices of the equatorial electrojet and counter-electrojet in the Indian region have been evolved based on certain reasonable assumptions, by a criterion depending on the difference in the horizontal field strength between a station under the electrojet axis (Trivandrum) and a station outside the jet influence (Alibag). The indices enable one to characterize each day of the year by an appropriate index. Distribution, in the months, seasons and over the years. of the electrojet and counter-electrojet frequencies at Trivandrum during a 20-year period. 1959-78, is examined. The salient features of the distributions are: (i) Strong electrojets (range $50 \mathrm{nT}$ ) and the afternoon counter-electrojets occur, on an average, on about $50 \%$ of the days in the year; (ii) For the counter-electrojet, the monthly frequencies show an annual variation with a summer maximum, and for the strong electrojet, a semi-annual variation with maxima in the equinoxes: (iii) A secondary maximum is noted in January in the occurrence frequency of both counter-electrojet and strong electrojet events; (iv) Intense electrojets occur with greater frequency in September.
\end{abstract}

Keywords. Electrojet; counter-electrojet; indices; frequency distribution; solar cycle modulation; lunar modulation.

\section{Introduction}

The equatorial electrojet, so named by Chapman (1951), is an intense electric current flowing east ward in the ionospheric E-layer in a narrow belt of latitudes centred on the dip equator. A depression in the daily variation of the horizontal component $(H)$ of the geomagnetic field below the night level, often observed at an observatory near the dip equator, has led to the concept of a current similar to the electrojet but with a flow in the reverse direction. Gouin and Mayaud (1967) have consequently called it the equatorial counter-electrojet. Some of the early studies on the counter-electrojet in the Indian region (the afternoon phenomenon) were based on the enumeration of the events by visual inspection of the magnetograms from Trivandrum (Sastri and Javakar 1972) and Kodaikanal (Rastogi 1973) situated slightly to the south and to the north respectively of the dip equator. Such an identification, however, becomes difficult on days other than relatively quiet days. In this paper, a method, based on some reasonable assumptions, is used to evolve indices characterizing each day by the strength of the electrojet and the counter-electrojet in the Indian region.

\section{Definition and derivation of the indices}

The method of evolving indices is similar to the one adopted earlier (Bhargava et al 1980) using hourly values of the horizontal intensity $H$ at Trivandrum (dip. lat. $-0.4^{\circ}$ ) near the axis of the equatorial electrojet and at Alibag (dip. lat. 12.8 ${ }^{\circ}$ ), an 
observatory outside the influence of the electrojet, both the stations differing only by about $4^{\circ}$ in longitude. The hourly $H$ values were first subjected to non-cyclic correction and the mean of 23 rd and 24 th hourly (local time) values were then subtracted to obtain the inequalities of the daily variation field at Trivandrum $(\Delta H T)$ and at Alibag $\left(\Delta H_{A}\right)$ above the night-time base at the respective stations. The twentyfour hourly differences ( $\Delta H T-\Delta H_{A}$ ) were then obtained for each day. The hourly differences around noon were considered for the definition of the electrojet index ( $E J$ index) and the differences in the afternoon hours ( 1200 to $1800 \mathrm{hr}$ local time) were used in defining the afternoon counter-electrojet index (CEJ index). The criterion for the classification of both the electrojet and counter-electrojet days is given in table 1 .

The assumptions underlying the above procedure are as follows: (i) The field of the extra-ionospheric currents can be considered the same at Trivandrum as at Alibag because of the proximity of the stations compared to the distance of the source.

During the disturbed days, small-period fluctuations (period $<60 \mathrm{~min}$ ) superımposed on the long period variations may not have phase synchronization at the two stations and also the effects of the fluctuating electric field of ionospheric origin may not be the same at Alibag and Trivandrum. These fluctuations would, however, be smoothed out to a large extent in the hourly mean values used in the analysis and the residual field is assumed to be not considerable. (ii) The field associated with the world-wide $S q$ currents at Alibag fairly represents the $S q$ field at Trivandrum. $S D$, index defined by Kane (1973) is based on such an assumption. Yacob (1977) finds that

Table 1. Definition of electrojet (EJ) and counter-electrojet (CEJ) indices

$$
\text { Classification (Index) }
$$

Condition

\section{Electrojet Index}

(Based on hourly differences, $\Delta H_{T}-\Delta H_{A}$, for 10-14 hr LT)

(0) All hourly values are negative

(1) Maximum value of $\Delta H_{T}-\Delta H_{A}$ is not equal to or greater than $25 \mathrm{nT}$

(2) Maximum value of $\Delta H_{T}-\Delta H_{A} \geqslant 25 \mathrm{nT}$ but $<50 \mathrm{nT}$

(3) Maximum value of $\Delta H_{T}-\Delta H_{A} \geqslant 50 \mathrm{nT}$ but $<75 \mathrm{nT}$

(4) Maximum value of $\Delta H_{T}-\Delta H_{A} \geqslant 75 \mathrm{nT}$ but $<100 \mathrm{nT}$

(5) Maximum value of $\Delta H_{T}-\Delta H_{A} \geqslant 100 \mathrm{nT}$

\section{Counter-electrojet index}

(Based on hourly differences, $\Delta H_{T}-\Delta H_{A}$, for 12-18 hr LT)

(0) $\Delta H_{T}-\Delta H_{A} \quad$ positive without a minimum in the afternoon hours.

(1) $\Delta H_{T}-\Delta H_{A} \quad$ positive but with a minimum in the afternoon hours.

(2) Minimum value of $\Delta H_{T}-\Delta H_{A}$ is not equal to or lesser than $-20 \mathrm{nT}$

(3) Minimum value of $\Delta H_{T}-\Delta H_{A}$ is $\leq-20 \mathrm{nT}$ but $>-40 \mathrm{nT}$

(4) Minimum value for $\Delta H_{T}-\Delta H_{A}$ is $\leq-40 \mathrm{nT}$

Note: In the case of counter-electrojet index, the minimum is identified when it is followed by an increase in $\Delta H_{T}-\Delta H_{A}$. 
to arrive at a suitable estimate of the electrojet field at Trivandrum for any hour, it would be sufficient to subtract, on the average, $\Delta H_{A}$ or at the most $1.1 \Delta H_{A}$ from $\Delta H_{T}$ for the corresponding hour. (iii) The excess field at Trivandrum is attributed to the electrojet effect. The range of $50 \mathrm{nT}$ of the field. which is nearly the diurnal range at Alibag, is treated as the normal electrojet effect because quiet-day range in $H$ at Trivandrum is found, on an average, to be twice the $H$ range at Alibag (Sastri and Jayakar 1970). Chapman (1951) states that the electrojet may be about double the normal current intensity over itšs narrow band.

\section{Analysis}

The 20-year period of the analysis, 1959-78, includes a complete solar cycle, viz. solar cycle 20 (1965-76). Each day during the period is characterized by an $\mathrm{EJ}$ index as well as a CEJ index by the criteria given in table 1 . The frequency distribution of the different indices is then calculated for each month. By an apprcpriate combination of these monthly data, the annual, seasonal and monthly frequencies for the whole period are obtained for each of the indices. Seasonal groupings are made into the three Lloyds' seasons: d - December solstitial season (November, December, January, February), $\mathrm{e}$-equinoctial season (March, April, September, October) and $\mathrm{j}$ - June solstitial season (May, June, July, August). Days with EJ indices 3, 4 and 5 which have, a round noon, a maximum hourly value of $\left(\Delta H_{T}-\Delta H_{A}\right)$ greater than $50 \mathrm{nT}$ are taken to represent, in that order, the days of strong electrojet. Days with CEJ indices 2, 3 and 4 only are counted as counter-electrojet days because these are the days which have at least one negative hourly value of $\left(\Delta H_{T}-\Delta H_{A}\right)$ in the afternoon hours.

\section{Results}

\subsection{General features}

The frequency distribution of different CEJ and EJ indices is given in table 2 for the entire 20-year period as well as in the three seasons. We note that the distribution of different CEJ and EJ indices is not uniform. CEJ indices 1 and 2 and EJ indices 2 and 3 occur more frequently than the other respective indices. The afternoon counterelectrojet events, represented by CEJ indices 2,3 and 4 , occur on an average on about $50 \%$ of the days. This increases to a little over $70 \%$ during the northern summer months. This is consistent with the earlier observation by Bhargava et al (1980) based

Table 2. Frequency distribution of different EJ and CEJ indices in the seasons.

\begin{tabular}{|c|c|c|c|c|c|c|c|c|}
\hline \multirow[b]{2}{*}{ Index } & \multicolumn{4}{|c|}{ Elect rojet (EJ) } & \multicolumn{4}{|c|}{ Counter-electrojet (CFJ) } \\
\hline & d & e & j & Total & d & e & j & Total \\
\hline 0 & 43 & 9 & 23 & 75 & 321 & 524 & 223 & 1068 \\
\hline 1 & 372 & 56 & 286 & 714 & 1153 & 849 & 460 & 2462 \\
\hline 2 & 954 & 404 & 926 & 2284 & 682 & 867 & 1291 & 2840 \\
\hline 3 & 700 & 975 & 860 & 2535 & 182 & 157 & 407 & 746 \\
\hline 4 & 244 & 706 & 287 & 1237 & 54 & 30 & 63 & 147 \\
\hline 5 & 75 & 280 & 60 & 415 & & & & \\
\hline Total & & & & 7260 & & & & 7263 \\
\hline
\end{tabular}


on a slightly smaller data-base and further strengthens their suggestion that the afternoon counter-electrojet variations need not be regarded as rare or abnormal but constitute one of the patterns of the normal daily variation. The maximum of CEJ occurence in summer ( $\mathrm{j}$-season) and the minimum in winter (d-season), noted earlier by Sastri and Jayakar (1972), is maintained in the total frequency of the three CEJ indices 2, 3 and 4. Strong CEJ events (CEJ indices 3 and 4), however, show a minimum in the equinoxes. Maximum number of very strong counter-electrojet (CEJ index 4) events occur in either of the solstitial seasons.

Strong electrojet (EJ indices 3, 4 and 5) occurs on about $55 \%$ of the days during the period of the analysis. Their frequency exhibits a strong bias towards the equinoxes which is even more pronounced in the case of EJ indices 4 and 5. During equinoxes, strong electrojets occur on nearly $80 \%$ of the days reducing to about $40 \%$ in winter. On any summer day strong $\mathrm{EJ}$ is as likely as a weak EJ.

\subsection{Frequency distribution in the months of the year}

The frequency of occurrence of CEJ and strong EJ over the months of the year during the period 1959-1978 is presented in figure 1 .

4.2a CEJ events: A broad dominant maximum around June-July and a secondary maximum in January in the occurrence frequency of CEJ conform to the known features of the annual progression of CEJ occurrence frequency (Bhargava and Sastri 1979). Such an annual variation is seen individually in the frequencies of the CEJ indices 2 and 3 . Although the two maxima are fairly evident, the annual variation is not well marked in the frequency distribution of the strong events (CEJ index 4). A feature noted in the CEJ frequency distribution over the months is that from April to September the highest frequency is registered for the CEJ index 2 , whereas for the rest of the months, October to March, index 1 has the maximum frequency. CEJ index 1
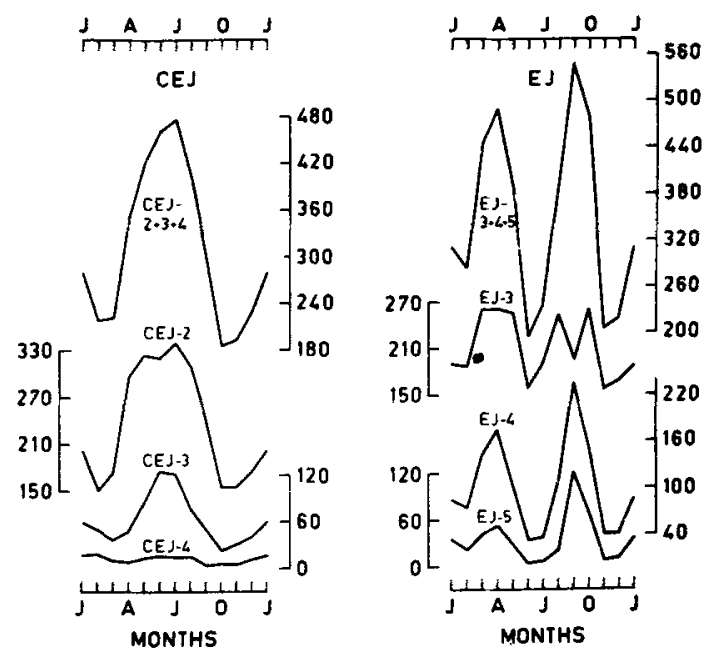

Figure 1. Frequency of occurrence of the afternoon counter-electrojet events (CEJ) and of the strong electrojet days (EJ: range $>50 \mathrm{nT}$ over the calendar months during the period 1959-1978. The distribution for each of the indices as well as for the three indices combined are shown separately. 
refers to events where, although there is no negative value, a minimum is still observed in the afternoon hours (table 1). Such cases are termed as partial counter-electrojet events (Rastogi 1974). Thus, in the summer months and a month on either side, the events which could be only partial events tend to register as weak counter-electrojets.

4.2b Strong Es events: The frequency distribution of the occurrence of strong electrojet ( $E$ indices 3,4 and 5 combined) is primarily semi-annual with the maxima in the equinoxes (figure 1). Surprisingly, a secondary peak in January, noticed in the CEJ frequencies, is a conspicuous feature in EJ occurrence also. Some features in the annual progression of the frequency of strong electrojet occurrences are as follows: (i) Strong electrojets are as few in the two summer months, June and July, as they are in the two winter months, November and December. (ii) There is an appreciable asymmetry in the occurrence of strong electrojets between the two equinoxes. Figure $1 \mathrm{~b}$, which illustrates the annual progression of frequency of EJ indices 3,4 and 5 individually, further shows equinoctial asymmetry is markedly large in the frequency of intense electrojets (EJ indices 4 and 5 ) with a peak in their occurrence in the month of September. This peak is accompanied by a pronounced 'dip' in the occurrence frequency of moderately strong electrojets (EJ index 3 ) in that month, compared to the frequency in the adiacent months, August and October.

\subsection{Lunar modulation in the occurrence frequency of $C E J$ and $\mathrm{EJ}$}

The distributions of the occurrence frequency of the counter-electrojet events (CEJ indices 2, 3 and 4 combined) and strong electrojet events (EJ indices 3, 4 and 5 combined) over the lunar phase are presented in figure $2 \mathrm{a}$ for the three seasons and for the year. Frequency distribution of each of the indices characterizing CEJ and strong $\mathrm{EJ}$, based on the data for the entire period, are shown separately in figure $2 \mathbf{b}$.

Lunar modulation is clearly seen in the frequency of both CEJ and EJ indices. The lunar semi-monthly wave in the occurrence frequency of CEJ with a maximum around the lunar phases 1 and 13 is consistent with the results of Sastri and Jayakar(1972) and Rastogi (1973). Curiously the lunar modulation shows a strong measure of dependence on season, being strong in winter and the equinoxes but very weak or nearly absent in summer. Lunar influence on the frequency of strong electrojet occurrence and its seasonal dependence are, in general, similar to what is observed in the counter-electrojet frequency. There is a perceptible phase shift in the variation of $\mathrm{EJ}$ frequency over lunar phase in the d-season as compared to that in the e-season. The lunar modulation is equally effective in the individual frequency distributions of each of the three $\mathrm{EJ}$ indices 3,4 and 5; but, in the case of CEJ indices lunar control decreases with increasing intensity of the counter-electrojet, almost becoming unimportant for the strong events (CEJ index 4).

\subsection{Distribution in the years}

Figure 3 gives the distribution of the occurrence frequency of strong electrojet days (EJ indices 3,4 and 5 combined) and of the counter-electrojet events over the years. Annual mean of the sunspot number $R_{z}$ and of the magnetic activity index $A_{p}$ are also included in the diagram. The occurrence frequency of strong electrojets runs parallel with the annual mean sunspot numbers. On the other hand, the maxima in CEJ occurrence are almost coincident with the minima in $R_{z}$; the minimum of occurrences 


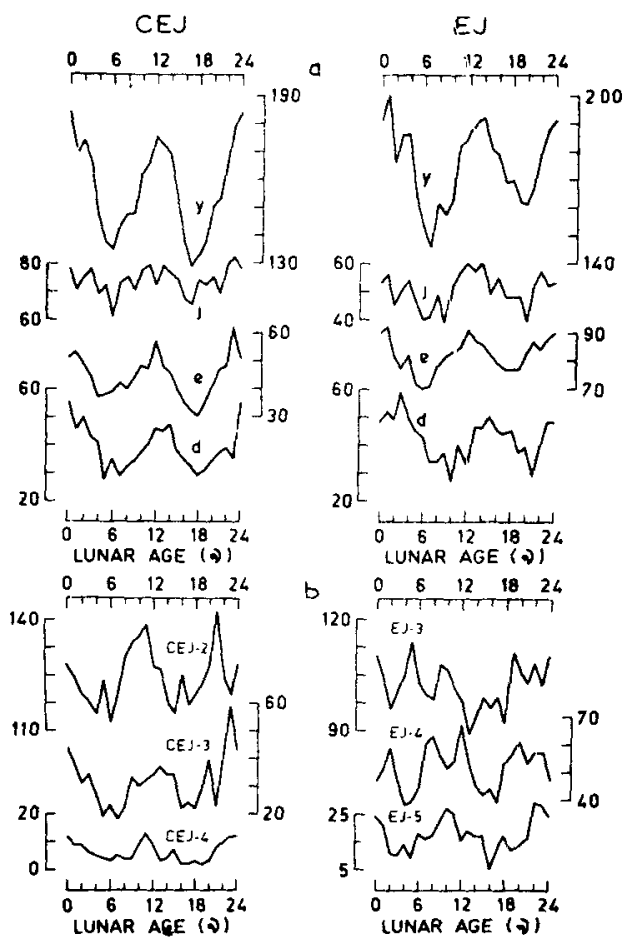

Figure 2. Distribution of the occurrence frequency, based on the data for the entire period 1959-78, of the afternoon counter-electrojet (CEJ indices 2, 3 and 4) and strong electrojet (EJ indices 3,4 and 5) events over the lunar phase (a) in the different seasons ( $d, e$ and $j$ ) and the year for the three indices combined, and (b) separately for each of the indices characterizing CEJ and strong EJ.

in 1968-69 is close to the broad maximum in $R_{z}$. Examining the association between the occurrence of counter-electrojet events, and the annual mean $A_{p}$ and $R_{z}$, Bhargava and Sastri (1979) found a precise anti-phase relationship between CEJ occurrences and $A_{p}$. The anti-phase relationship observed in the present results (figure 3 ) is not that close, particularly in the later part of the period studied. Though the average level of geomagnetic activity generally varies with the sunspot cycle, the pattern shows differences from one cycle to another. It has been suggested (Gosling et al 1977) tha solar cycle 20 is unusual in that a very large peak has occurred in 1973-75, 5-6 years after the sunspot maximum which can be seen from the plot of $A p$ in figure 3 . As the 436 events examined earlier by Bhargava and Sastri (1979) have been identified by visual inspection of the Trivandrum magnetograms, it is likely that, with the increase in geomagnetic activity, many of the CEJ events would have been obscured resulting in a low CEJ frequency coincident with the $A_{p}$ peak in the declining phase of the solar cycle. Because of the objective criterion adopted here, many of the events which have not been accounted for earlier are included in the present enumeration. CEJ frequency shows, as noted earlier, an almost inverse relationship with $R_{z}$. This is in agreement with the results of Marriott et al (1973) for Huancayo and Rastogi (1974) for Kodaikanal, Addis Ababa and Huancayo.

To examine whether the level of magnetic activity as characterized by the daily magnetic activity index, $A_{p}$, has any control on the CEJ occurrence. the CEJ occurrence 

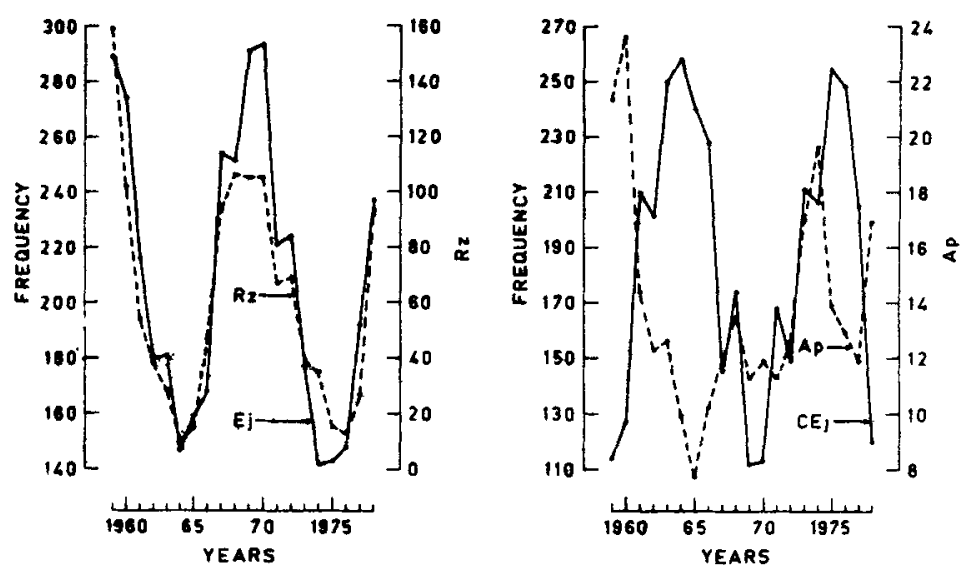

Figure 3. Frequency of occurrence of the afternoon counter-electrojet events (CEJ indices 2, 3 and 4 combined) and of strong electrojet days (E.J indices 3, 4 and 5 combined) over the years from 1959 to 1978 . The variation in the annual mean relative sunspot number $R_{z}$ and the annual mean magnetic activity index $A_{p}$ are also included.

frequency is computed separately for days with $A_{p} \leqslant 7$ and days with $A_{p}>7$, representing respectively the quiet and disturbed conditions. The frequencies, obtained for the entire period as well as for the three seasons separately, are given in table 3 expressed as percentages of the total number of days in the whole period and in the respective seasons. In the 20 -year period, days with $A_{p} \leqslant 7$ are about $35 \%$ of all days. Among such quiet days, CEJ occurs on $52 \%$ of the days. This percentage remains the same for the disturbed days $\left(A_{p}>7\right)$ as well as for all the days. Percentages, similarly calculated, separately for each season also remain more or less the same, except for a small rise in the $\mathrm{j}$-season percentage for the quiet days. It thus appears that the nature of the day, quiet or disturbed, does not make a difference in the CEJ occurrence. The near-antiphase relationship between the annual mean $A p$ and the annual CEJ occurrence frequency, seen in figure 3, may be more apparent than real. It could simply be a consequence of the strong association between the solar and magnetic activities on a long-term scale.

\section{Discussion}

As the solar and lunar days are incommensurable, the pattern of geomagnetic lunar daily variation $L$ at any station progressively changes from one solar day to a nother. The magnitude of $L$ is the largest in winter and the smallest in summer; equinoctial values tend to be close to the winter values. The variation of lunar effect at any solar hour shows two high (positive) and two low (negative) values indicating the dominance of the lunar fortnightly tide (Rao 1972). The lunar phases with maximum occurrence of EJ correspond to the lunar phase when the fortnightly tide attains maximum magnitude around the local noon. Since the counter-electrojet effect is observed as a depression in the daily variation of $H$, the lunar phases with maximum CEJ occurrence correspond to the lunar phases with maximum negative effects in the fortnightly tide at about the solar hour 15 when the occurrence of the afternoon counter-electrojet is found to be most frequent. Also, seasonal variation of the lunar 
Table 3. Occurrence frequency of the afternoon counter-electrojet event in the Indian region during quiet $(A p \leqslant 7)$ and disturbed $(A p>7)$ days (percentages)

\begin{tabular}{|c|c|c|c|c|}
\hline \multirow[t]{2}{*}{ CEJ occurrence among days } & \multicolumn{3}{|c|}{ Seasons } & \multirow[t]{2}{*}{ Year } \\
\hline & d & e & j & \\
\hline With $A p \leqslant 7$ & 36 & 42 & 86 & 52 \\
\hline With $A p>7$ & 40 & 44 & 70 & 51 \\
\hline All & 38 & 43 & 72 & 51 \\
\hline 7 in all days & 37 & 31 & 36 & 35 \\
\hline
\end{tabular}

modulation on the occurrence frequency of CEJ as well as EJ is in accord with the seasonal variation in the strength of $L$, being maximum in winter and minimum in summer. It thus appears that the nature of the lunar modulation and its seasonal dependence is consistent with that resulting from the distortion of the normal solar daily variation by the regular lunar daily variation. This view gains further support from the fact that a shift of two lunar phases in the time of maximum effect of lunar fortnightly tide at noon during the equinoxes, relative to the winter months, has resulted in a similar shift in the occurrence maximum of strong EJ over the lunar phases.

The strong lunar modulation seen in the CEJ occurrence has led to the suggestion that, on the average, the counter-electrojet event results from the superimposition of the lunar tidal wave on the solar daily variation of $H$ appropriate for the concerned longitude as well as the epoch of the year and of the solar cycle (Rastogi 1975). However, we note that even in the winter months, when the lunar influence is the strongest, CEJ events that occur irrespective of the lunar phase are half as many as the events that occur in favourable lunar phases and the frequency of such events has a distribution in seasons similar to that of the total frequency. Further, if CEJ occurrence were solely due to the lunar perturbation one would expect the maximum number of events in the winter months and a minimum in the summer months when the lunar effects are small. But a contrary feature is observed in the occurrence frequency of CEJ over the months where summer months show large frequencies. It has been earlier noted (Sastri and Jayakar 1972; Forbes and Lindzen 1976) that when the afternoon CEJ events occur on a series of consecutive days, the minimum usually occurs around the same solar hour. Thus the results favour the earlier conclusion of Rastogi (1973) that the Moon's effect is not to cause but only to modulate the occurrence of the counter-electrojet, rather than his suggestion that CEJ events are of lunar tidal origin (Rastogi 1975). Mayaud (1977) also makes a similar remark in regard to the lunar influence on the equatorial CEJ occurrence. Nevertheless, it is possible that the spurt in the occurrence frequency of CEJ as well as strong EJ in January compared to the other winter months may be related to the lunar effect because $L$ has been shown to undergo a strong global enhancement in January (Schlapp and Malin 1979).

The genesis of the equatorial electrojet is fairly understood. But the possible sources of the equatorial counter-electrojet still remain obscure. Examining the pattern of magnetic variations associated with a counter-electrojet along a chain of stations on the same longitude Stening (1977) observes that in addition to winds associated with the semi-diurnal tidal mode, the effects due to planetary waves on occasions seem more likely to be responsible for deviations from the normal pattern. The occurrence 
of CEJ during magnetically quiet days is explained by Raghavarao and Anandarao (1980) as due to the vertical winds of gravity wave origin, which with a proper choice of their latitudinal extent and magnitude could account for the different intensities and widths of the CEJ observed on different days. Rastogi and Patel (1975) and Rastogi (1981) show that a number of counter-electrojet events are associated with the rapid changes of the latitude $(\theta)$ of the interplanetary magnetic field (IMF) from southward to northward direction, in the presence of large arid rather steady value of the scalar field $(B)$ which produces pure electric fields that would be anti-parallel to the $S q$ field at the magnetic equator. Such events have their signatures as abrupt and short-period variations in $H$ at the equatorial stations over an ex tended longitude zone and also at non-equatorial stations and occur at the same un iversal time, Rastogi (1980) thus suggests a lunar tidal source as well as a magnetospheric source for the CEJ. However, the feature, that the afternoon minimum is found at about the same solar hour when CEJ occurs on a succession of relatively quiet days, remains unexplained.

The strength of the equatorial electrojet is known to undergo a strong semi-annual variation with maxima in the equinoxes, which is ascribed (Tarpley 1973) to the equatorward movement of the northern and souther'n current foci in September and March respectively. The equinoctial maxima are we:l reflected in the pattern of the frequency distribution of strong EJ. The approach of the focus towards a low latitude station outside the influence of the electrojet would, however, cause a decrease in the $S q$ amplitude. Such a decrease in the amplitude of the daily variation of the horizontal field in September was clearly noticed by Sastri et al (1978) in the annual progression of the monthly amplitudes at Alibag. The strength of the electrojet is evaluated here by the difference in the $H$ field at a station under the electrojet (Trivandrum) and one outside its influence (Alibag). As a consequence electrojets of weak or moderate strength around September would tend to be enhanced in their strength to be registered as strong electrojets. This would result in a higher frequency for the strong electrojets and a reduced number for the weak electrojets in September, a feature well brought out in the frequency distributions of $E$ indices 3,4 and 5 .

A close parallelism between the occurrence of strong electrojets and sunspot number is consistent with the hypothesis that intensity of the electrojet increases with increasing solar activity, a behaviour well observed and reported.

In view of the poor understanding of the possible sources of counter-electrojet at present, it is difficult to explain the inverse relationship between the cownter-electrojet occurrence and $R_{z}$. However, the possibility of CEJ-related current distorting the electrojet current to the extent of its reversal is large when normal electrojet current is weak, as during the sunspot minimum.

In respect of the seasonal and solar cycle changes in the occurrence of counterelectrojet, particularly intense CEJ, the behaviour is similar to that of the occurrence of abnormal quiet days (AQD) reported by Arora (1972) and Sastri and Murthy (1978). $A Q D$ are defined (Brown and Williams 1969) as quiet days on which the maximum of the daily variation in $H$ occurs outside the normal interval, taken to be $0830-1330 \mathrm{Lr}$. Discussing the possible causative mechanisms for AQD Brown (1975) suggests an atmospheric origin for this abnormality and relates it to the influence of the upward propagating planetary waves from the troposphere and stratosphere into the ionospheric dynamo region.

\section{Conclusion}

The solar cycle, lunar phase as well as seasonal distributionis of the occurrence 
frequency of the afternoon counter-electrojet events (CEJ) at Trivandrum, identified by an objective criterion, broadly conform to the known characteristics of the CEJ occurrence in the Indian region. The primary features of the seasonal and solar cycle control of strong electrojets (EJ) is also consistent with the known regular behaviour of the electrojet. Thus, the criteria based on which the CEJ and EJ events a re identified and categorized by the respective indices appear valid indicating that the underlying assumptions in deriving the indices are fairly reasonable. Prominent features of the frequency of occurrence of the afternoon counter-electrojet events and the strong electrojets at Trivandrum during the 20-year period 1959-78 can be summarized as follows:

(i) Strong electrojets (range $>50 \mathrm{nT}$ ), like the afternoon counter-electrojet events, occur, on an average, on about $50 \%$ of the days in the year.

(ii) While summer (j-season) has the highest frequency of CEJ events (in about $70 \%$ of the days) strong EJ occurs most in the equinoxes (on about $80 \%$ of the days).

(iii) The variation in the occurrence frequency of CEJ is primarily annual and of strong EJ is clearly semi-annual with equinoctial maxima.

(iv) A secondary maximum in January, noted earlier in the CEJ frequency, is also seen in the occurrence frequency of strong electrojets although EJ frequency has minima in the solstitial months.

(v) Very intense electrojets (EJ indices 4 and 5) occur more frequently.in September which month consequently shows a highly reduced percentage of weak electrojets ( $E J$ indices 1 and 2).

\section{Acknowledgements}

One of the authors (BNB) wishes to thank the Director, Indian Institute of Geomagnetism, Bombay, for providing facilities and data and to the Council of Scientific and Industrial Research, Government of India, for financial assistance.

\section{References}

Arora B R 1972 Indian J. Meteorol. Geophys. 23195

Bhargava B N and Sastri N S 1979 Geomagn. Geoelectr. 3197

Bhargava B N, Sastri N S, Arora B R and Rajaram R 1980 Ann. Geophys. 36231

Brown G M 1975 J. Atmos. Terr. Phys. 37107

Brown G M and Williams W R 1969 Planet. Space Sci. 17455

Chapman S 1951 Arc. Meteorol. Geophys. Bioklim Series A Band IV 368

Forbes J M and Lindzen R S $1976 \mathrm{~J}$. Atmos. Terr. Phys. 38911

Gosling J T, Asbridge J R and Bame S J 1977 J. Geophys. Res. 823311

Gouin P and Mayaud P N 1967 Ann. Geophys. 2341

Kane R P 1973 J. Atmos. Terr. Phys. 351565

Marriott R T, Schieldge J P, Venkateswaran S V and Cain J C 1973 The quiet-time equatorial counterelectrojet, Symposium on low level satellite surveys (Second General Scientific IAGA Assembly. Kyoto, Japan)

Mayaud P N 1977 J. Atmos. Terr. Phys. 391055

Raghavarao R and Anandarao B G 1980 Geophys. Res. Lett. 7357

Rao D R K 1972 Pure Appl. Geophys. 95131

Rastogi R G 1973 Planet. Space Sci. 211355

Rastogi R G 1974 J. Geophys. Res. 791503

Rastogi R G 1975 Proc. Indian Acad. Sci. A81 80

Rastogi R G 1980 Indian J. Radio Space Phys. 101

Rastogi R G and Patel V L 1975 Proc. Indian Acad. Sci. A82 121 
Sastri J H and Murthy B S 1978 Indian J. Radio Space Phys. 762

Sastri N S and Jayakar R W 1970 Indian J. Meteorol. Geophys. 21279

Sastri N S and Jayakar R W 1972 Ann. Geophys. 28589

Sastri N S, Arora B R and Rao D R K 1978 Ann. Geophys. 34325

Schlapp D M and Malin S R C 1979 Geophys. J. R. Astron. Soc. 59161

Stening R J 1977 J. Almos. Terr. Phys. 391071

Tarpley J D 1973 J. Aimos. Terr. Phys. 351063

Yacob A 1977 J. Atmos. Terr. Phys. 39601 